\title{
Texture features extraction of multi-coloured fancy yarn
}

DOI: 10.35530/IT.071.06.1728

\section{ABSTRACT - REZUMAT}

\section{Texture features extraction of multi-coloured fancy yarn}

In the present work, a framework of extracting important texture features of multi-coloured fancy yarns is proposed. A self-developed image capturing apparatus is used to record the image of fancy yarn. Subsequently, the captured digital images are processed to produce spatially corresponding pixel points to reconstruct digital images of the object, including image greying, filtering and morphology processing. At last, different segmentation methods are used to extract the texture features of fancy yarns, and the optimal segmentation method to each kind of fancy yarn is analysed.

Keywords: fancy yarn, digital image analysis, image segmentation, colour textures, image graying

Determinarea caracteristicilor de textură ale firelor de efect multicolore

În lucrarea de față, este propusă o metodă de determinare a caracteristicilor importante de textură ale firelor de efect multicolore. Un echipament de captare a imaginii este utilizat pentru a înregistra imaginea firelor de efect. Ulterior, imaginile digitale captate sunt procesate pentru a produce puncte pixel corespunzătoare spațial, pentru a reconstrui imaginile digitale ale obiectului, incluzând scara de gri, filtrarea și procesarea morfologică a imaginii. În cele din urmă, sunt folosite diferite metode de segmentare pentru a determina caracteristicile de textură ale firelor de efect și se analizează metoda optimă de segmentare a fiecărui tip de fir de efect.

Cuvinte-cheie: fir de efect, analiza imaginii digitale, segmentarea imaginii, texturi colorate, scara de gri a imaginii

\section{INTRODUCTION}

Yarn is the basic unit of fabrics. The knowledge of texture feature of yarn is essential for reconstructing the simulated appearance of the fabric. In particular, the multi-coloured fancy yarns, which include a wealth of texture information, such as yarn twist, yarn fineness, surface brightness and colour alteration, are recently emerged as new building blocks for the multi-colour fabric $[1,2]$. The exaction of the texture features of fancy yarn dramatically benefits the simulation of appearance streaks in fabrics [3, 4].

There are two ways to obtain yarn image, i.e., experimental measurement and computer simulation. There are advantages and disadvantages to each method. If the yarn characteristics, such as colour, illumination and evenness, can be accurately measured, the simulated appearance of the fabric is close to that of a real product. However, it requires arduous and delicate pre-treatment on yarn surface due to excessive hairiness and unevenness in practical textile production and thus may lower the work efficiency of the designer. In contrast, one can easily set up the basic parameters of the yarn using computer simulation, which does not require any pre-treatment of yarn. However, computer simulation generally yields unreliable results due to the lack of an adequate way to extract texture characteristics of the actual yarn, leading to the unrealistic appearance of the simulated fabric. Therefore, it is essential to establish a framework to extract the texture features in various kinds of multi-coloured fancy yarns.
In the present work, a framework of extracting important image features through a serial of image processing techniques is demonstrated. It eliminates or reduces the background noises of the image to the maximum $[5,6]$, whereas the colour texture features and detailed information about yarn structure such as yarn diameter and yarn evenness are retained. At last, the effects of different segmentation algorithms on fancy yarn images are compared and analysed, and optimal algorithm of segmenting different types of fancy yarn images is proposed.

\section{THEORETICAL MODELS}

For multi-colour fancy yarn images, segmentation algorithms are needed to extract the textural properties, that is, information on the evenness, colour and diameter of the coloured spun yarn. Several image segmentation methods are employed in an attempt to find the optimal one that is suitable for extracting textural features of fancy yarn.

\section{OTSU threshold segmentation algorithm}

The OTSU method, which is named after Nobuyuki Otsu and also known as the maximum inter-class variance method, is a self-adaptive thresholding method used to determine the threshold values for binary image segmentation. The OTSU method was based on grayscale properties of an image where inter-class variance increases while intra-class variance decreases between image background and object. In other words, the method can be used to 
maximize the separation between foreground and background for the purpose of highlighting the object region from the background [7-9], based on the assumption that the mixture density function of object foreground and background in an image is comprised of two sub-distributions that conform to normal distributions with equal variances.

The OTSU thresholding segmentation algorithm can automatically and rapidly calculate the threshold values based on the grey values of a grey scale image to maximize the grey value differences between foreground and background regions, thereby obtaining a binary image with clearly distinguishable foreground and background.

Assuming the size of an image is $M \times N$ pixels and the number of the image's different grey levels is $L$, that is $[0,1,2, \ldots, L-1]$. The number of pixels in $i^{\text {th }}$ grey level is $n_{i}$; the threshold value for segmentation of foreground and background is denoted by $T(k)=k$; the pixels in the image whose grey values fall within $[0, k]$ are classified as $C_{1}$; and the pixels in the image whose grey values fall within $[k+1, L-1]$ are classified as $C_{2}$. Given the presence of components in a normalized histogram, the procedure of the algorithm is shown in the following equation (1) and equation (2):

$$
\begin{gathered}
q_{i}=\frac{n_{i}}{M N} \\
\sum_{i=0}^{L-1} q_{i}=1
\end{gathered}
$$

where $q_{i}$ is the percentage of pixels in $i^{\text {th }}$ grey level. With the known threshold value $k$, then the probability that the pixels are classified to $C_{1}$ is $P_{1}(k)$, while the probability that they are classified to $C_{2}$ is $P_{2}(k)$.

$$
\begin{gathered}
P_{1}(k)=\sum_{i=0}^{k} q_{i} \\
P_{2}(k)=\sum_{i-k-1}^{L-1} q_{i}=1-P_{1}(k)
\end{gathered}
$$

Therefore, the average gray value of pixels classified to $C_{1}$ is $m_{1}(k)$, as shown in equation (5). Similarly, the average gray value of pixels classified to $C_{2}$ is $m_{2}(k)$, as shown in equation (6) [7].

$$
\begin{gathered}
m_{1}(k)=\sum_{i=0}^{k} i P\left(\frac{i}{C_{1}}\right)=\frac{1}{P_{1}(k)} \sum_{i=0}^{k} i q_{i} \\
m_{2}(k)=\sum_{i-k-1}^{L-1} i P\left(\frac{i}{C_{2}}\right)=\frac{1}{1-P_{1}(k)} \sum_{i-k-1}^{L-1} i q_{i}
\end{gathered}
$$

The final inter-class variance is $g$, as shown in equation (7) [10]. The threshold value $T(k)$ that maximizes the interclass variance can thus be solved iteratively [11].

$$
g=P_{1} P_{2}\left(m_{1}-m_{2}\right)^{2}
$$

\section{Kmeans segmentation algorithm}

As a typical clustering algorithm based on distance partition, the Kmeans algorithm uses distance evaluation indicators as measures of similarities between pixel samples $[12,13]$. Essentially, Kmeans algorithm is an iterative algorithm that tries to partition data and seeks an optimal solution to the clustering criterion function of each partitioned cluster, thereby maximizing intra-cluster similarity and the inter-cluster difference between the partitioned data clusters. The letter $\mathrm{K}$ represents the centre value of an arbitrarily selected initial cluster, that is, the cluster value; while Means denotes the mean value, that is, the mean value of all sample values in each partitioned cluster, which represents the centre of the boundary of each cluster.

An image is partitioned into several clusters, and the initial centroid of each cluster should be present when using the Kmeans clustering segmentation algorithm. Therefore, selecting the number of clusters and initial cluster centres is crucial for the goodness of clustering. The selected number of clusters should be in line with the best partitioning of samples. Otherwise, a different number of clusters may result in totally different clustering results and increase the processing time. Therefore, data partitioning is required for each iteration of the algorithm to ensure the data are as close to each cluster as possible. When all data are included in its cluster, one round of iterative process is completed, and new centroids will replace the old ones. If clustering results do not change at the next iteration, the convergence is achieved for the clustering. The specific procedure of the algorithm is shown as follows:

I. Given the known training-data object $\left\{x^{1}, x^{2}, \ldots, x^{p}\right\}$ is an element of $R_{n}$, where $p$ vectors are partitioned into $i$ groups, the clustering centre of each group of vectors is obtained to ensure that the distance criterion function which serves as the evaluation indicator is minimized. Select $k$ data objects as initial cluster centres, that is, $\mu_{1}, \mu_{2}, \ldots, \mu_{k}$;

II. Calculate the Euclidean distance of each data object from each cluster centre, denoted by $D^{(i)}$, as shown in equation (8):

$$
D^{(i)}=\operatorname{argmin}\left\|x_{i}-\mu_{j}\right\|^{2}
$$

where $\mu_{j}$ is the centroid of $x^{i}$ 's cluster.

III. After completing the first round of data object clustering, perform recalculations to update the centre of each cluster to ensure data objects can be continuously clustered based on new cluster centres, as shown in equation (9) [13].

$$
\mu_{j}=\frac{\left.\sum_{i}^{p} x^{(i)}\right|_{D^{(i)}=j}}{\left.\sum_{i}^{p} 1\right|_{D^{(i)}=j}}
$$

IV. Repeat the above procedure until there are no changes in cluster centres, that is, the distortion function $J(D, \mu)$ in equation (10) converges, and the sum of squares of all data objects' Euclidean distances from their cluster centres is obtained.

$$
J(D, \mu)=\sum_{i=1}^{p}\left\|x^{(i)}-\mu_{D^{(i)}}\right\|^{2}
$$

\section{Mean-shift segmentation algorithm}

The Mean-shift algorithm is widely used for clustering, image smoothing, segmentation and video tracking. In image segmentation, Mean-shift is a statistical iterative algorithm that estimates the kernel density $[14,15]$. The specific procedure of the algorithm is as follows: Assume there are $n$ data points in a $d$-dimensional space, the set of data points is $\left\{x_{1}, \ldots, x_{j}\right\}$, where 
$i$ denotes $1,2, \ldots, n$. Select a random point $x$ in the space; the mean-shift vector $M_{h}(x)$ is basically defined by equation (11), where $S_{h}(x)$ is the high latitude region with a radius $h$, as shown in equation (12) [16], where $k$ denotes the number of observations falling within the range of $S_{h}(x)$.

$$
\begin{gathered}
M_{h}(x)=\frac{1}{k} \sum_{x_{i} \in S_{h}(x)}\left(x_{i}-x\right) \\
S_{h}(x)=\left\{y:\left(y-x_{i}\right)^{T}\left(y-x_{i}\right)<h^{2}\right\}
\end{gathered}
$$

After incorporating the Gaussian kernel function, the Mean-shift algorithm, i.e., equation (11), is converted into density estimator, as shown in equation (13), where $k(x)$ denotes the Gaussian kernel function, $c_{k, d} / n^{h d}$ is unit density. To find the points of the maximum probability density, the equation (14) can be derived from equation (13).

$$
\begin{gathered}
f_{h, k(x)}=\frac{c_{k, d}}{n h^{d}} \sum_{i=1}^{n} k\left(\left\|\frac{x-x_{i}}{h}\right\|^{2}\right) \\
\hat{\nabla} f_{h, k(x)}=\frac{2 c_{k, d}}{n h^{d+2}} \sum_{i=1}^{n}\left(x-x_{i}\right) k^{\prime}\left(\left\|\frac{x-x_{i}}{h}\right\|^{2}\right)
\end{gathered}
$$

Let $g(x)=-k^{\prime}(x)$, then equation (15) can be obtained.

$$
\begin{gathered}
\hat{\nabla} f_{h, k(x)}=\frac{2 c_{k, d}}{n h^{d+}} \sum_{i=1}^{n} g\left(\left\|\frac{x-x_{i}}{h}\right\|^{2}\right) . \\
\cdot\left[\frac{\sum_{i=1}^{n} x_{i} g\left(\left\|\frac{x-x_{i}}{h}\right\|^{2}\right)}{\sum_{i=1}^{n} g\left(\left\|\frac{x-x_{i}}{h}\right\|^{2}\right)}-x\right]
\end{gathered}
$$

Finally, a mean-shift vector $m_{h, g(x)}$ can be obtained, as expressed by equation (16). If $\hat{\nabla} f_{h, k(x)}=0$, the maximum value, that is, regions of maximum densities can be obtained if and only if $m_{h, g(x)}=0$. Thus, the equation of new origin coordinates, as shown in equation (17), is obtained.

$$
\begin{gathered}
m_{h, g(x)}=\frac{\sum_{i=1}^{n} x_{i} g\left(\left\|\frac{x-x_{i}}{h}\right\|^{2}\right)}{\sum_{i=1}^{n} g\left(\left\|\frac{x-x_{i}}{h}\right\|^{2}\right)}-x \\
x=\frac{\sum_{i=1}^{n} x_{i} g\left(\left\|\frac{x-x_{i}}{h}\right\|^{2}\right)}{\sum_{i=1}^{n} g\left(\left\|\frac{x-x_{i}}{h}\right\|^{2}\right)}
\end{gathered}
$$

\section{METHODOLOGY}

\section{Image capture}

The recordation of high-quality yarn images is the basis for the simulation of weft knitted fabrics. In the present work, an image capturing apparatus developed by the research institute of textile technology in Jiangnan University [17], as shown in figure 1, is used to record the yarn image. The colour images are recorded onto a single high-resolution colour charge-coupled device (CCD) sensor and saved as the BMP or TIF or JPG image formats. The output

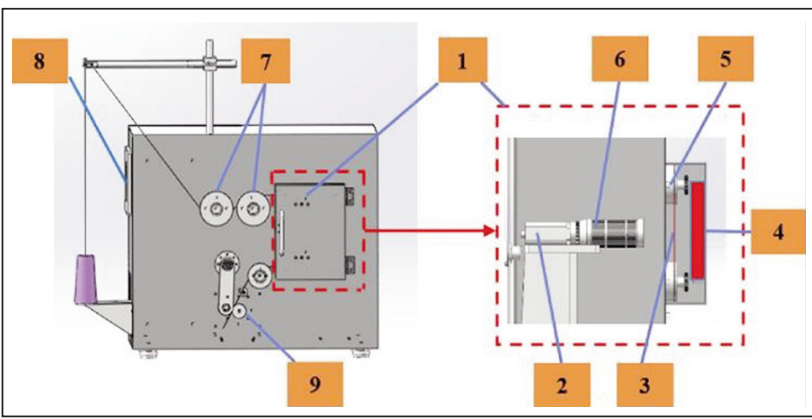

Fig. 1. Yarn image acquisition device: 1 - imaging box; 2 - CCD area image sensor; 3 - yarn; 4 - a light source; 5 - yarn guide device; 6 - camera lens; 7 - tension control panel; 8 - touch screen; 9 - Servo motor for output roll

image size is $56 \times 473$ pixels, and the physical size of the image is calibrated to $1 \mathrm{~cm}$. When recording images, the yarn is fully straightened out under the camera. The camera is set up in such a way that the main feature of fancy yarn is fully exposed under the camera. This setup ensures the accuracy of image processing later on. The device can measure yarn diameter and colour distribution along the longitudinal direction of moving fancy yarn to extract texture features of yarn.

\section{Image graying}

The grey scale processing is to convert a colour image into a grayscale image. The colour of each pixel is composed of Red (R), Green (G) and Blue (B) components, and the value ranges of the three components are between 0 and 255, which means each pixel has more than 10 million kinds of colours. The grey scale image is a special colour image with equal proportions between $\mathrm{R}, \mathrm{G}$ and $\mathrm{B}$, i.e., the variation range of each pixel is only between 0 and 255 . There are no colour changes in the grey scale image except the brightness value, which significantly reduces the amount of computation in post-processing and improves the processing efficiency. In this paper, the weighted average method, as shown in equation (18), is used to deal with yarn image:

$$
H=R \times W_{r}+G \times W_{g}+B \times W_{b}
$$

where $W_{r}, W_{g}, W_{b}$ are weight ratios of $R, G$ and $B$ components; $H$ is the tristimulus value.

\section{Image filtering}

Image filtering is used to enhance some spatial frequencies of an image to improve the grey difference between target and background. In this work, two smoothing filters were employed, i.e., linear filtering and median filtering to process the images of fancy yarns. The linear filter replaces each pixel value of image with the average value of a surrounding pixel field, which is also called the average field method. The median filter is a nonlinear method, similar to convolution. Instead of a weighted sum, the grey value of the middle pixel point of the pixel field is used. Thus, the median filter can eliminate isolated 
noise pixels. Meanwhile, the details, such as edge characteristics, are well reserved.

\section{Binarization of image and morphology processing}

Even after the filter processing, the noises and concave/convex edges of the image cannot be entirely removed, which is problematic for the subsequent fabric simulation. Therefore, the image is turned into the binary representation to prepare for morphology processing. In general, the binarization of the image divides the grey values of all pixel points in the image into two categories: black and white, i.e., the background and the target, respectively. The goal is to classify the 256 brightness levels in the grayscale image by comparing with thresholds, so that the pixels can be divided two fields, which are the background field and the foreground field (l.e., the target), respectively. In general, grey value greater than or equal to the threshold value belongs to the foreground field, and their grey values are set as 255; however, If the grey value is less than the threshold, these pixels are excluded from the object area (i.e., background field), and their grey values are set as 0 . Mathematical morphology is an accurate tool for image analysis of geometrical structures, such as size, shape and convexity [18], based on the binary image. There are four basic operations, i.e., dilation, erosion, opening and closing [18], among which the opening operation can remove the sharp objects in images. We use the opening operation of mathematical morphology to process the binary representation of yarn, as shown in figure 2. The sharp edges (marked by a rectangular circle) in figure $2, a$ is removed in figure $2, b$ after opening operation. The image processing, including greying, filtering, morphology processing and segmentation, were carried out using the self-developed code written in MATLAB software package.

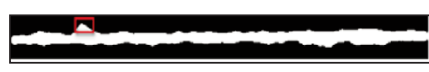

a



b
Fig. 2. Binary image of yarn: $a-$ image before opening operation; $b$ - image after opening operation

\section{RESULTS AND DISCUSSION}

Figure 3 shows the recorded images of different types of fancy yarns, which are later used for investigating the effects of different segmentation methods on multi-coloured fancy yarn. Figure 4 shows the images processed by using two different filtering methods. The images processed with linear filter include more fuzziness, hairness and noises than the images processed with the median filter. Therefore, we chose the median filter to process the recorded images of fancy yarns.

In order to find out the optimal segmentation methods to these images, the fancy yarn images are processed by using three segmentation methods and then compared with recorded images. To characterize the differences between the segmented and recorded
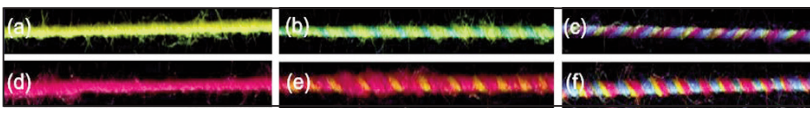

Fig. 3. Recorded images of fancy yarns: a - singlecoloured yarn; $b$ - two-coloured yarn with the 3:7 weight ratio between blue and yellow fiber components; $c$ - three-coloured yarn with the 2:4:4 weight ratio between red, yellow and blue fiber components; $d$ - singlecoloured slub yarn; $e$ - two-coloured slub yarn with the 1:1 weight ratio between yellow and red fiber components; $f$ - three-color slub yarn with the 1:1:1 weight ratio between red, yellow and blue fiber components

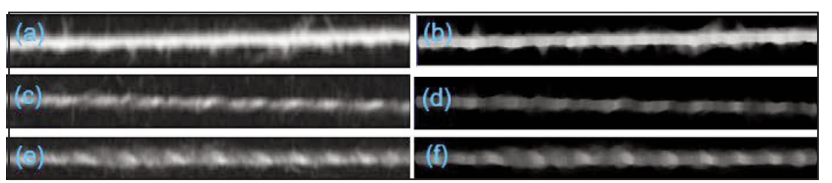

Fig. 4. Comparison of filtering methods of grayscale images of yarns: $a, c, e-$ images processed with the linear filter method; $b, d, f$-images processed with the median filter method

images, each image is decomposed into $R, G$ and $B$ colour channels respectively. Then, the mean value and standard deviation (SD) of each channel are calculated, respectively, as shown in table 1.

Figure 5, $a, b, g, h, m, n$ show the segmented images of the single-coloured fancy yarn by using different segmentation methods. It can be seen that there are no differences in three segmentation methods concerning the extraction of texture features, such as yarn evenness and colour; however, they are slightly different regarding the edges. Figure 5,g retains edge characteristics of the yarn compared to Figure 5, a. Our observation is further verified by comparing the mean values and SDs between segmented and recorded images in table 1 , in which the mean values and SDs based on Kmeans method are closer to those of recorded images than the other two methods. In Figure 5, $m$, the appearance of yarn is more evident than others, and there is no hairiness. In terms of segmentation time, Kmeans clustering segmentation is quicker than OTSU threshold segmentation and mean-shift segmentation; OTSU threshold segmentation is quicker than mean-shift segmentation.

Figure $5, c, d, i, j, o, p$ shows the segmented images of two-coloured fancy yarn with different segmentation methods. Through comparing with recorded images, i.e., figure $4, a, f$, the segmented images of figure $5, o, p$ are closest ones, which indicates meanshift algorithm is better at processing two-coloured fancy yarn images than the other two methods, and is consistent with the mean values and SDs in table 1. However, in terms of segmentation time, OTSU segmentation is quicker than Kmeans clustering segmentation and mean drift segmentation, which means the Kmeans method becomes slower than the OTSU method when processing two-coloured fancy yarn images. The decrease of efficiency of Kmeans method is attributed to the increased number of images, the differences in colour appearances between the target and the background, and the 


\begin{tabular}{|c|c|c|c|c|c|c|c|c|c|c|c|c|c|c|c|c|c|c|}
\hline \multirow{2}{*}{$\frac{\mathscr{d}}{\frac{\mathscr{c}}{0}}$} & \multicolumn{3}{|c|}{$\begin{array}{l}\text { Mean } \\
\text { (OTSU) }\end{array}$} & \multicolumn{3}{|c|}{$\begin{array}{c}\text { SD } \\
\text { (OTSU) }\end{array}$} & \multicolumn{3}{|c|}{$\begin{array}{c}\text { Mean } \\
\text { (Kmeans) }\end{array}$} & \multicolumn{3}{|c|}{$\begin{array}{c}\text { SD } \\
\text { (Kmeans) }\end{array}$} & \multicolumn{3}{|c|}{$\begin{array}{c}\text { Mean } \\
\text { (Mean-shift) }\end{array}$} & \multicolumn{3}{|c|}{$\begin{array}{c}\text { SD } \\
\text { (Mean-shift) }\end{array}$} \\
\hline & $\mathrm{R}$ & G & B & $\mathrm{R}$ & G & B & $\mathrm{R}$ & G & B & $\mathrm{R}$ & G & B & $\mathrm{R}$ & G & B & $\mathrm{R}$ & G & B \\
\hline $1 \#$ & 48.9 & 52.2 & 12.5 & 86.2 & 91.3 & 42.8 & 51.1 & 54.7 & 11.8 & 82.1 & 87.7 & 22.1 & 57.8 & 60.8 & 23.9 & 91.1 & 95.6 & 55.3 \\
\hline $2 \#$ & 64.2 & 12.6 & 30.7 & 95.6 & 13.1 & 41.6 & 63.4 & 19.49 & 25.9 & 91.5 & 40.0 & 54.4 & 58.1 & 6.84 & 24.6 & 90.8 & 15.1 & 42.3 \\
\hline $3 \#$ & 63.4 & 15.2 & 16.1 & 88.9 & 29.5 & 16.3 & 63.0 & 27.4 & 29.5 & 92 & 57.1 & 55.1 & 83.4 & 15.3 & 25.9 & 88.7 & 30.0 & 26.3 \\
\hline 4\# & 37.9 & 52.4 & 25.2 & 60.5 & 70.6 & 49.3 & 39.8 & 55.9 & 25.9 & 66.6 & 85.1 & 51.3 & 37 & 50.8 & 24.9 & 60.8 & 80.7 & 42.8 \\
\hline $5 \#$ & 26.0 & 23.5 & 29.6 & 54.4 & 52.8 & 59.4 & 25.3 & 23.0 & 28.9 & 54.2 & 52.6 & 59.1 & 21.8 & 18.3 & 27.0 & 41.4 & 38.3 & 48.4 \\
\hline $6 \#$ & 43.6 & 29.9 & 33.0 & 76.8 & 61.6 & 61.5 & 56.2 & 38.4 & 44.5 & 87.5 & 73.6 & 76.1 & 54.5 & 41.2 & 44.4 & 81.0 & 64.6 & 67.5 \\
\hline \multirow{6}{*}{ 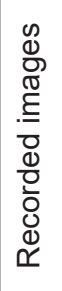 } & 1\# & $2 \#$ & $3 \#$ & $4 \#$ & $5 \#$ & $6 \#$ & $1 \#$ & 2\# & $3 \#$ & $4 \#$ & $5 \#$ & $6 \#$ & $1 \#$ & $2 \#$ & $3 \#$ & $4 \#$ & $5 \#$ & $6 \#$ \\
\hline & \multicolumn{6}{|c|}{$\mathrm{R} /$ mean } & \multicolumn{6}{|c|}{ G/mean } & \multicolumn{6}{|c|}{ B/mean } \\
\hline & 55.4 & 75.0 & 82.0 & 34.2 & 22.7 & 59.8 & 60.4 & 22.3 & 22.3 & 51.3 & 19.3 & 41.3 & 13.4 & 24.6 & 24.6 & 20.7 & 28.4 & 47.6 \\
\hline & \multicolumn{6}{|c|}{$\mathrm{R} / \mathrm{SD}$} & \multicolumn{6}{|c|}{ G/SD } & \multicolumn{6}{|c|}{$\mathrm{B} / \mathrm{SD}$} \\
\hline & 1\# & $2 \#$ & $3 \#$ & $4 \#$ & $5 \#$ & $6 \#$ & $1 \#$ & 2\# & $3 \#$ & $4 \#$ & $5 \#$ & $6 \#$ & $1 \#$ & $2 \#$ & $3 \#$ & $4 \#$ & $5 \#$ & $6 \#$ \\
\hline & 80.3 & 90.0 & 87.9 & 57.1 & 42.0 & 80.0 & 85.5 & 39.9 & 46.2 & 78.5 & 38.7 & 64.5 & 22.8 & 52.2 & 43.6 & 36.4 & 48.6 & 66.7 \\
\hline
\end{tabular}
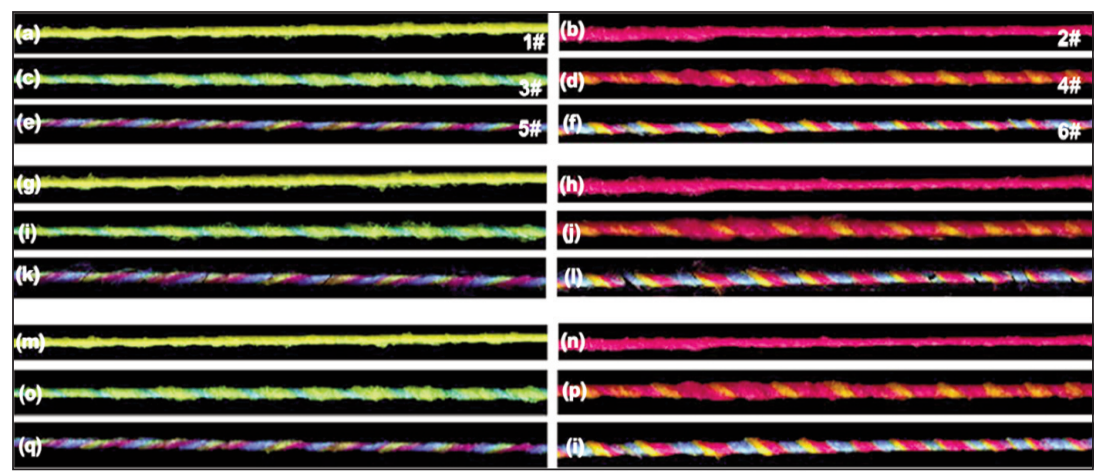

Fig. 5. Segmented images of single-coloured, two-coloured and three-coloured fancy yarns by using three different image segmentation methods: $a-f-$ segmented images generated by using the OTSU segmentation method; $g-l-$ segmented images generated by using the Kmeans segmentation method; $m-r$ - segmented images generated by using the mean-shift segmentation method spikes appear at the edges, which may change the yarn diameter. However, in the case of the meanshift method, the pixel points of the edges of the images are appropriately processed, and thus, it is better than the OTSU method in terms of segmenting three-coloured fancy yarn. This is also consistent with the mean values and SDs in table 1.

\section{CONCLUSIONS}

In this study, we successfully developed a framework of extracting the texture features of multicoloured fancy yarn. The high-resolution images of fancy yarns are number of iterations, which jointly increase the processing times of Kmeans method [19-21].

Figure $5, e, f, k, l, q, r$ shows the segmented images of three-coloured fancy yarn with different segmentation methods. The effect of Kmeans clustering segmentation is not ideal, because, for three-coloured fancy yarn, some colours are close to the background colour, leading to the discontinuity in the segmented image, as shown in figure $5, k, I$. However, for the OTSU and mean-shift segmentation methods, there are no discontinuities in the segmented images owning to different processing techniques. It appears that the images processed by OTSU and mean-shift methods are similar to one another, and the only difference occurs to the edges. For the OTSU method, recorded by using a self-developed image capturing device. The captured digital images are first converted by image greying, and subsequently, processed with filtering. We found the images processed with a median filter include less fuzziness, hairness and noises than the images processed with a linear filter. Furthermore, using the opening operation of mathematical morphology, we remove the sharp objects in the images. At last, we compare and analyse different segmentation methods to extract the texture features of digital images of fancy yarn. It is found that the Kmeans method is more effective in segmenting single-coloured and two-coloured fancy yarns, while the mean-shift method is more effective in segmenting three-coloured fancy yarn.

\section{REFERENCES}

[1] Guo, M., Sun, F., Gao, W., Theoretical and experimental study of color-alternation fancy yarns produced by a double-channel compact spinning machine, In: Textile Research Journal, 2019, 89, 14, 2741-2753 
[2] Yang, R.H., Xue, Y., Gao, W.D., Airflow characteristics of different groove type during rotor spinning process, In: Industria Textila, 2017, 68, 3, 165-169.

[3] Kliman, H.L., Pike, R.H., Method of simulating by computer the appearance properties of a fabric, USA, US4984181A, 1991

[4] Magnenat-Thalmann, N., Luible, C., Volino, P., Lyard, E., In From measured fabric to the simulation of cloth, 2007 10th IEEE International Conference on Computer-Aided Design and Computer Graphics, IEEE, 2007, 7-18

[5] Yeung, K., Li, Y., Zhang, X., Yao, M., Evaluating and predicting fabric bagging with image processing, In: Textile Research Journal, 2002, 72, 8, 693-700

[6] Behera, B., Image-processing in textiles. In: Textile Progress, 2004, 35, 2-4, 1-193

[7] Otsu, N., A threshold selection method from gray-level histograms, In: IEEE transactions on systems, man, and cybernetics, 1979, 9, 1, 62-66

[8] Rosenfeld, A., Digital picture processing, Academic press, New York, USA, 1976, 22-24

[9] Pal, N.R., Pal, S.K., A review on image segmentation techniques, In: Pattern recognition, 1993, 26, 9, 1277-1294

[10] Liu, D., Yu, J, In Otsu method and K-means, In: Ninth International Conference on Hybrid Intelligent Systems, IEEE, 2009, 344-349

[11] Huang, L.K., Wang, M.J.J., Image thresholding by minimizing the measures of fuzziness, In: Pattern recognition, 1995, 28, 1, 41-51

[12] Jain, A.K., Data clustering: 50 years beyond K-means, In: Pattern recognition letters, 2010, 31, 8, 651-666

[13] Han, J., Pei, J., Kamber, M., Data mining: concepts and techniques, Elsevier, MA, USA, 2011, 34-35

[14] Comaniciu, D., Meer, P., Mean shift: A robust approach toward feature space analysis, In: IEEE Transactions on Pattern Analysis \& Machine Intelligence, 2002, 5, 603-619

[15] Leibe, B., Leonardis, A., Schiele, B., Robust object detection with interleaved categorization and segmentation, In: International journal of computer vision, 2008, 77, 1-3, 259-289

[16] Fukunaga, K., Hostetler, L., The estimation of the gradient of a density function, with applications in pattern recognition, In: IEEE Transactions on information theory, 1975, 21, 1, 32-40

[17] Li, Z., Pan, R., Zhang, J., Li, B., Gao, W., Bao, W., Measuring the unevenness of yarn apparent diameter from yarn sequence images, In: Measurement Science and Technology, 2015, 27, 1, 015404

[18] Coster, M., Chermant, J.L., Image analysis and mathematical morphology for civil engineering materials, In: Cement and Concrete Composites, 2001, 23, 2-3, 133-151

[19] Arthur, D., Vassilvitskii, S, In How slow is the k-means method?, In: Symposium on computational geometry, 2006, $1-10$

[20] Har-Peled, S., Sadri, B., How fast is the k-means method?, In: Algorithmica, 2005, 41, 3, 185-202

[21] Scott, A.J., Knott, M., A cluster analysis method for grouping means in the analysis of variance, In: Biometrics, $1974,507-512$

Authors:

\section{Dr. PENG CUI, Dr. YUAN XUE}

Key Laboratory of Eco-Textiles, Jiangnan University,

1800 Lihu road, Binhu district, Wuxi, 214121, Jiangsu, China

e-mail: cuipengdhu@gmail.com

Corresponding author:

YUAN XUE

e-mail: fzxueyuan@qq.com 